\title{
Philosophiques
}

\section{D’une lecture coupable de tout ce qu'elle laisse choir...}

À propos de Freud et le problème de la culpabilité, par Ghislain

\section{Charron}

\section{Lise Monette}

Volume 7, numéro 2, octobre 1980

URI : https://id.erudit.org/iderudit/203146ar

DOI : https://doi.org/10.7202/203146ar

Aller au sommaire du numéro

Éditeur(s)

Société de philosophie du Québec

ISSN

0316-2923 (imprimé)

1492-1391 (numérique)

Découvrir la revue

Citer cet article

Monette, L. (1980). D'une lecture coupable de tout ce qu'elle laisse choir... À propos de Freud et le problème de la culpabilité, par Ghislain Charron.

Philosophiques, 7(2), 377-382. https://doi.org/10.7202/203146ar d'utilisation que vous pouvez consulter en ligne.

https://apropos.erudit.org/fr/usagers/politique-dutilisation/ 


\title{
D'UNE LECTURE COUPABLE DE TOUT CE QU'ELLE LAISSE CHOIR . . .*
}

\author{
À propos de Freud et le \\ problème de la culpabilité \\ par Ghislain Charron, Ottawa, \\ Éditions de l'Université d'Ottawa, 1979, 200 p.
}

par Lise Monette

1. LA PHILOSOPHIE (LA THÉORIE) DONT ON PART MARQUE DE SON SCEAU L'OBJET D'ÉTUDE

La philosophie est définie à quelques reprises par G. Charron comme une "étude des essences". Disons-le tout net : je ne partage pas cette vision classique. La philosophie, dans son projet épistémologique, me semble être ce qui lui permet d'articuler et de ne point nier ses rapports avec (la) les sciences qui lui sont contemporaines. Tributaire et critique de ces dernières, elle se doit de travailler à repérer ce qu'il en est des éléments idéologiques et scientifiques qui les habitent (ce que Charron tente de faire dans la dernière partie de son livre pour la théorie freudienne). Mais, de plus, toute philosophie, pensée, naît (n'est) d'une pratique (pédagogique ${ }^{1}$, politique, clinique, etc.). Or, ce livre situe et discute la métapsychologie freudienne dans ses rapports à la clinique, mais qu'en est-il de la pratique de l'auteur lui-même?

\section{PEUT-ON ÊTRE PSYCHANALYSTE ET PHILOSOPHE EN MÊME TEMPS ?}

G. Charron tranche la question d'entrée de jeu, trop rapidement, oserais-je dire. L'ambiguïté n'en demeure pas

\footnotetext{
* Version, non modifiée, du commentaire présenté au congrès de l'Acfas, à Québec, en mai 1980.

1. Octave Mannoni, Un commencement qui n'en finit pas, Éd. du Seuil, 1980.
} 
moins, et ses traces sont immédiatement repérables dans la facture même de l'ouvrage qui repose la question évitée sur le plan épistémologique. En effet, le point de départ de cette recherche est constitué de matériel dit clinique. Le problème est double. Y a-t-il de la clinique autre que la sienne, i.e. celle dans laquelle nous sommes partie prenante dans le processus transférentiel ? Comment faut-il qualifier un matériel clinique de «seconde main », quel est son statut? La position stratégique des premiers chapitres, leur place privilégiée leur font occuper la fonction "d'entités empiriques". En sont-ils pour les lecteurs que nous sommes? Le matériel clinique se présente ici comme "support " de l'analyse et de la preuve. Ne joue-t-il pas le même rôle que celui que Freud assignait à la "réalité historique"?

3. Remarques décousues - À défaut d'être cousues DE FILS BLANCS - SUR LE DOUTE, LA MORT ET LA DETTE CHEZ L'OBSESSIONNEL

Ce travail sur le sentiment de culpabilité repose de façon prévilégiée sur le cas de "l'homme aux rats». Il y est fait souvent mention de son ambivalence vis-à-vis de son père, bien que ce concept comme tel ne soit pas développé dans le volume. L'auteur insiste, à la suite de Freud, sur la désintrication des pulsions chez ce type de patient. Par ailleurs, la polarisation de l'analyse sur le père laisse dans l'ombre la place et la fonction de la mère dans une telle configuration. Serge Leclaire, dans Démasquer le réel ; un essai sur l'objet en psychanalyse (Éd. du Seuil, 1971), jette quelques lumières sur ces questions. Il illustre, à l'aide d'un exemple clinique, comment la mère, au moment de la phase oedipienne, donne satisfaction aux désirs de l'enfant plutôt que de le frustrer, c'est-à-dire qu'elle maintient le leurre qu'il est l'objet unique de son désir. Autrement dit, la fonction maternelle demeure symbiotique, pourrait-on dire; elle se fige, se cramponne dans son rôle d'étayage de la pulsion et évacue son rôle de médiatrice, de «porte-parole» de la loi. La mère se présente ainsi comme but et objet du désir de l'enfant, s'accapare tout l'investissement libidinal et bloque le déplacement, la déviation vers le père. La mère doit être l'index qui pointe la loi dont le père 
réel n'est que le support. Cette loi peut alors être comprise comme celle justement de la dérive nécessaire du désir plutôt que sa prohibition, qui référerait à une phase ultérieure, postérieure à l'Oedipe. Cette « gélification du devenir »chez l'obsessionnel trouverait là sa source. La dure règle de la nécessité à laquelle il soumet toutes ses activités (loisir autant que travail) les présentifie sous le mode du besoin (Sisyphe condamné à sa tâche) et témoigne de cette fixation initiale dont il n'a pu se déprendre, à une étape où objet de besoin et objet de désir coexistaient.

Dépasser le père pour le fils se pose en termes cedipiens chez Freud, écrit G. Charron (p. 72). Si on émet l'hypothèse - aisément vérifiable par la clinique - que dans le cas de la fille, elle y met en jeu son identité, c'est-à-dire que nous nous trouvons devant un problème antérieur à l'Oedipe, à savoir que la composante narcissique prend le pas sur la composante libidinale objectale, on peut alors se demander si ce facteur n'a pas été escamoté chez le fils. On pourrait ainsi comprendre l'importance de la dette chez l'obsessionnel, dette à liquider minutieusement dans la vie quotidienne, solde non liquidable d'une retenue de la mère dans l'imaginaire. L'obsessionnel est coupable, complice, victime consentante et d'autant plus coupable de ce gel de l'échange symbolique. Le sentiment de culpabilité précéderait la reconnaissance de la "faute originelle », originaire, celle de la mère.

Le doute, le mouvement perpétuel d'oscillation, symptôme de l'ambivalence des sentiments de l'obsessionnel, peuvent aussi se lire, dans cette perspective, comme formation réactionnelle à l'immobilisme qui le fait piétiner sur place. Leclaire écrit : " la structure obsessionnelle peut être conçue en tant que refus redoublé de la possibilité ultime de sa propre mort " (p. 138). L'obsessionnel se vit sans avenir, tout est "déjà fini ", joué. Leclaire ajoute que son patient, en philosophe, lui dit : "Je voudrais quand même retrouver la possibilité d'utiliser toutes mes possibilités. "Coupable, il l'est déjà d'avoir fait le "mort ", c'est-à-dire d'avoir évité toute confrontation de ses désirs avec le père (précisons plutôt que la confrontation n'a pas eu lieu, car il ne s'agissait pas de 
ses désirs mais de ceux de sa mère) ; coupable, il le deviendra en tentant d'échapper à la règle maternelle.

Cette « construction» met-elle en question le sentiment de culpabilité en tant « qu'angoisse devant la perte d'amour» (p. 98) ? ${ }^{2}$ Dans un premier temps, nous semblons être en présence d'un «trop plein d'amour ». Faut-il y voir l'apparition de l'angoisse comme signal de l'ambivalence, c'est-à-dire de la tentative d'échapper à l'emprise de la figure maternante avec les conséquences que cela impliquerait? La connexion entre cette angoisse et l'angoisse sociale ( $\mathrm{p} .98)^{3}$ mérite réflexion. Nommons clairement l'enjeu : le conservatisme sur le plan politique trouverait-il ici quelques racines?

Ce qui précède met surtout en évidence ce que, à défaut d'un meilleur terme, j'appellerai le refoulement "primaire " chez l'obsessionnel de la tendance tendre et que recouvre le refoulement «secondaire» de la tendance sadique. Nous retrouverions ici les deux niveaux du sentiment de culpabilité inconscient dont il est question dans ce livre.

\section{NOUS SOMMES TOUS FILS DE KAFKA ...}

Au sujet de l'affirmation de G. Charron que les «questions de Freud sur le sentiment de culpabilité ne sont pas philosophiques ${ }^{4}$, il serait intéressant d'articuler Kant et Freud par rapport à Lacan à propos de la loi et de sa transgression. Chez Lacan, la transgression de la loi se fait à l'intérieur de l'ordre symbolique. À la limite, elle le renforcit. Le « hors-la-loi », c'est le psychotique pour qui le « Nom-duPère $»$ est demeuré forclos : le réel fera alors retour sur le mode hallucinatoire. Ce qui nous pousse à nous interroger ici, c'est une théorie qui fait l'économie du sentiment de culpabilité. Lacan, tout comme Deleuze dans Présentation de Sacher-Masoch, le froid et le cruel (Union générale d'éditions, 1967), souligne comment la loi, lorsqu'elle ne se fonde plus sur le Bien, devient forme pure, pure indétermination. Deleuze écrit «Elle

\footnotetext{
2. Charron, G. Freud et le probleme de alpabilité, Éditions de l'Université dOttawa, Ottawa, 1979 .

3. Idem.

4. Idem, p. 119.
} 
définit un domaine d'errance où l'on est déjà coupable, i.e. où l'on a déjà transgressé les limites avant de savoir ce qu'elle est : ainsi l'Oedipe » (84). Lacan, dans les Écrits, avait déjà indiqué que la loi est identique au désir refoulé. Si le désir ne peut s'éprouver qu'en se soumettant à la loi . . . du désir devenu méconnaissable, Deleuze nous indique une voie explicative de la nature inconsciente du sentiment de culpabilité en démontrant que le masochisme repose sur un double processus de dénégation de la castration et de désexualisation des représentations.

\section{L'INTERPRÉTATION : ENTRE LE DISCOURS PERFORMATIF ET LA CROYANCE}

La construction en analyse n'a rien à voir avec la prédiction. G. Charron l'indique avec netteté. Faut-il pour autant soutenir qu'elle est "reconstruction de l'histoire vécue " ou encore "reconstitution d'un sens perdu"? Ceci relève d'une approche herméneutique dont les racines remontent à l'interprétation des textes sacrés. Ricœur en retrace la filiation dans son livre sur Freud. Mais ce dernier, bien qu'il se réfere à l'archéologie et qu'il ait cherché à vérifier historiquement le bien-fondé de ses "construits", définit plutôt la construction à partir de trois effets :

a) rendre compte, intégrer le plus grand nombre possible d'événements, de fantasmes et de souvenirs du patient dans une scène, une "mise en scène " à valeur historique qui ne peut se ramener au concept d'explication ou d'élucidation. En outre, à cause de la connotation intellectuelle de ces référents ;

b) faire que ce «dire» de l'analyste soit performatif, c'est-à-dire qu'il transforme, réorganise, affecte et représente ;

c) produire un « construit » qui entraîne l'adhésion, la croyance.

Jean-Luc Donnet ${ }^{5}$ distingue trois types de croyance : la première s'appuie sur la logique de la preuve ; la seconde sur l'expérience vécue, c'est-à-dire la réalité perceptive, et la

5. J.-L. Donnet, "Une croyance à l'œuvre " in Nouvelle Revue de Psychanalyse, $\mathrm{n}^{\circ} 18$, automne 1978, Gallimard. 
troisième sur la soumission à l'attente du maître. La dynamique du processus analytique ne relève ni de la première ni de la troisième. Dans une note, Donnet cite Winnicott pour préciser ce dont il s'agit : " il faut pour l'analyse savoir attendre que tous les événements du passé puissent être vécus comme des projections. " 6

Si je suis d'accord avec G. Charron quand il affirme que la psychanalyse n'est pas une connaissance qui s'élabore par système mais en référence à un modèle, ceci ne résout pas tous les problèmes. Car, me semble-t-il, «l'entité empirique» dans ce cas-ci est le fantasme, et "l'entité épistémologique» est une "fiction théorique ", comme le développe Maud Mannoni dans son dernier volume ${ }^{7}$.

Département de philosophie

Université du Québec à Montréal

6. Idem, p. 235.

7. Maud Mannoni, La théorie comme fiction : Freud, Groddeck, Winnicott, Lacan, Éd. du Seuil, 1979. 